\title{
PROBABILITY ESTIMATION ON THE RISK OF MORTALITY OF PATIENTS WITH END-STAGE RENAL DISEASE
}

\author{
JACKIE D. URRUTIA, JAYA C. DE GUZMAN', AARON VITO M. BAYGAN², EDCON B. BACCAY², LINCOLN A. \\ BAUTISTA $^{2}$
}

Polytechnic University of the Philippines-Parañaque Campus Parañaque City, Philippines

Email: jackieurrutia20@gmail.com

Received: 25 Apr 2016 Revised and Accepted: 27 May 2016

\begin{abstract}
The kidneys perform life-maintaining functions like monitoring and regulating body fluids. These excrete excess fluids from the body and retain the substances necessary for the body's continuing function. If the kidneys malfunction, it may cause some kidney complications that could lead to EndStage Renal Disease (ESRD) or worst, it may lead to death. End-Stage Renal Disease (ESRD) or $5^{\text {th }}$ stage chronic kidney failure is a growing health condition problem that continues to rise in the Philippines. Kidney Diseases especially End Stage Renal Diseases is the $7^{\text {th }}$ leading cause of death in the country. Using Logistic Regression analysis, this study analyzed the record data such as the age, gender and the complications of 247 patients with Pulmonary Congestion and/or Cardiovascular disease diagnosed with ESRD at M. V. Makabali Memorial Hospital from the year 2008-2013. The present study was undertaken to identify the risk factors which causes to increase the death probability of an ESRD patient. The research concluded that pulmonary congestion and cardiovascular disease are significant factors that increase the patients' probability to encounter death. Moreover, an ESRD patient with cardiovascular disease has a higher chance to encounter death with a probability of $68.52 \%$ than an ESRD patient with pulmonary congestions with a probability of $57.79 \%$. The results also revealed that if a patient suffered from both complications, he/she has a higher risk of mortality with a probability of $98.6 \%$. ESRD may not be curable but with the proper management of health condition and maintaining a healthy lifestyle, it can be prevented and possibly could lessen the risk of death.
\end{abstract}

Keywords: Cardiovascular Disease, ESRD, Logistic Regression, Probabilities, Pulmonary Congestion

(C) 2016 The Authors. Published by Innovare Academic Sciences Pvt Ltd. This is an open access article under the CC BY license (http://creativecommons.org/licenses/by/4.0/) DOI: http://dx.doi.org/10.22159/ijpps.2016v8s2.15208.

\section{INTRODUCTION}

Kidneys perform life-maintaining functions like monitoring and regulating body fluids. These excrete excess fluids from the body and retain the substances necessary for the body's continuing function. The kidneys perform the essential function of removing waste products from the blood and regulating the water fluid levels [1]. If the kidneys malfunction, it may cause some kidney complications that could lead to End-Stage Renal Disease (ESRD). Having Kidney disease can also increase one's chances of having heart and blood vessels disease. It can also affect the other parts of the body like a sudden rise in potassium levels in your blood (hyperkalemia) for example, which could impair your heart's ability to function and may be lifethreatening. Other effects include having weak bones and increased risk of bone fractures, fluid retention and fluid accumulation in the lungs (pulmonary edema) [2]. Kidney disease is usually progressive. It typically does not reach the end stage until 10 to 20 y after diagnosis with chronic kidney disease which may also develop slowly.

ESRD is when kidneys are functioning below 10 percent of their normal, and they can no longer support a person's day-to-day life. At this stage, the kidneys can no longer remove waste and/or excess water from the patient's body [3]. It is also called the $5^{\text {th }}$-stage Chronic Kidney failure which is a health problem that continues to escalate in the country every year. Further, some factors considerably cause to decrease the patients' possibility to survive ESRD like Cardiovascular Disease or Pulmonary Congestion. Cardiovascular disease is the major killer in ESRD. Cardiovascular death risk is at least an order of magnitude higher in ESRD patients, even after adjusting age and status. Cardiac failure is a rapidly lethal condition among ESRD patients which appears to mediate much of the adverse prognostic impact of ischemic heart disease. Many risk factors act in concert to produce cardiovascular disease in ESRD. Many which can be treated, suggest that the huge burden of disease can be reduced considerably [4]. Dialysis can be a very common treatment for patients with ESRD. A person with ESRD has a severe reduction in kidney function. Various complications may appear at this stage and pulmonary congestions may be one of the possible consequences. The kidneys of ESRD patients cannot remove waste effectively, resulting to excess fluid build-up, and overloaded pulmonary congestions. Another problem for ESRD patients is that dialysis treatment may not be the best choice. When condition develops to ESRD, various toxins and wastes may build up in the patients' blood while dialysis can only help eliminate micro molecular toxins like creatinine, urea, and uric acid [5].

Last year, the National Kidney and Transplant Institute (NKTI) reported that kidney diseases, especially End-Stage Renal Disease (ESRD), are the $7^{\text {th }}$ leading cause of death in the country [6]. This is often associated to, Filipinos love for eating foods that are flavorful and rich but are high in sodium and preservatives that can cause damage to the kidneys. This unhealthy lifestyle of Filipinos causes the Department of Health $(\mathrm{DOH})$ to be alarmed over the soaring cases of kidney failure specially End-Stage Renal Disease in the country.

It is for the cited conditions that the researchers resolved to conduct a probability estimation of patients with ESRD. Using Logistic Regression Analysis that is widely used in medical research, the study purported to identify the patients' age, gender, health status and complications suffered particularly pulmonary congestions or cardiovascular disease. The intended output is a Logistic modelodds and probability of death among patients with ESRD.

\section{Objective of the study}

Through the use of Logistic Regression Analysis, the main focus of this study is to determine if the following prevalent factors: cardiovascular disease, pulmonary congestions, age, and gender have significant effect that causes to increase of death probability of a patient, as well as to compute the patients' probability of death caused by ESRD. Also, to generate the odds and probabilities of the End-stage Renal Disease patients. Furthermore, the study aims to make a comparison of the generated odds to know the difference of ESRD patients possessing any given covariates or combinations of it, to the condition of an ESRD patient with no other complications. 


\section{Research paradigm}

The researchers analyzed all the data obtained from the records of M. V. Makabali Memorial hospital with the following diagnoses: ESRD with cardiovascular disease, ESRD with pulmonary congestion, age and gender of the patients through the use of Logistic Regression Analysis specifically the forward stepwise method to obtain only the significant factors and to come up with a model that generates the odds and probabilities of patients with ESRD.

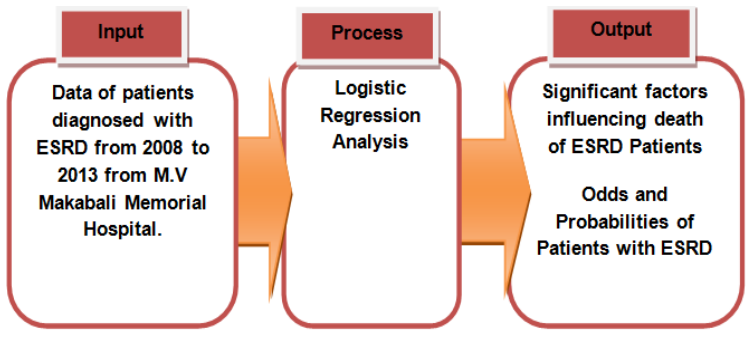

\section{Statement of the problem}

This research aims to estimate the odds and probability of an individual patient with ESRD. Also, it sought to answer the following questions:

1. What is the risk profile of patients with ESRD?

2. What are the significant risk factors that cause to increase the death probability of an ESRD patient?

3. What would be the logistic regression model of ESRD patients?

4. What are probabilities of patients diagnosed with the following factors:

5. What are the odds of an ESRD patient with and without complications?

a. Cardiovascular disease;

b. Pulmonary congestions; and

c. Both with cardiovascular disease and pulmonary congestions?

6. What are the Odds Comparison given the covariates? Or its combinations?

\section{Scope and limitations}

The data are obtained from the records compiled by the physicians and experts from M. V. Makabali Memorial Hospital with a total of 247 patients. These patients have chronic kidney disease and are diagnosed with End-Stage Renal disease from 2008 to 2013. The data gathered consists of ESRD with Cardiovascular disease, ESRD with Pulmonary Congestion, age, gender and status of the patients. The patients' statuses were observed to compute the probability of death among ESRD patients.

\section{Significance of the study}

The findings of the study will be helpful in determining the odds and probabilities of an ESRD patients and this study will be beneficial specifically in educating ESRD patients about the possible events that would happen if they have the said disease. Also, the outcome of this study will be significant in knowing the complications that must be avoided before the development of the symptoms emerge.

\section{MATERIALS AND METHODS}

\section{Population and covariates}

End Stage Renal Disease patients were recorded by their age, gender, and their ailments namely: Cardiovascular Disease or Pulmonary Congestion, diagnosed within the span of five years, precisely from 2008 until 2013. The data was gathered from M. V. Makabali Memorial Hospital with 247 total number of ESRD patients.

\section{Logistic regression}

The study used logistic regression which calculates the probability of an event occurring over the probability of an event not occurring, and the impact of independent variables is usually explained in terms of odds. With logistic regression, the mean of the response variable $p$ in terms of an explanatory variable $x$ is modeled relating $p$ and $x$ through the equation $p=\alpha+\beta x$. With logistic regression we model the natural log odds as a linear function of the explanatory variable:

$\operatorname{logit}(\mathrm{y})=\ln ($ odds $)=\ln \left(\frac{\mathrm{p}}{1-\mathrm{p}}\right)=a+\beta_{1} \chi_{1}+\beta_{2} \chi_{2}+.+\beta_{k} \chi_{k}$

Where $p$ is the probability of interested outcome and $x$ is the explanatory variable. The parameters of the logistic regression are $\alpha$ and $\beta$. This is the simple logistic model. Taking the antilog of equation (1) on both sides, one can derive an equation for the prediction of the probability of the occurrence of interested outcome as:

$$
\begin{aligned}
\mathrm{p}=\mathrm{P}\left(\mathrm{y} \mid \mathrm{x}_{1}, \mathrm{x}_{2}, \mathrm{x}_{3}, \cdots \mathrm{x}_{\mathrm{k}}\right) & =\frac{\mathrm{e}^{\mathrm{a}+\beta_{1} \mathrm{x}_{1}+\beta_{2} \mathrm{x}_{2}+\cdots+\beta_{\mathrm{k}} \mathrm{x}_{\mathrm{k}}}}{1+\mathrm{e}^{\mathrm{a}+\beta_{1} \mathrm{x}_{1}+\beta_{2} \mathrm{x}_{2}+\cdots+\beta_{\mathrm{k}} \mathrm{x}_{\mathrm{k}}}} \\
= & \frac{1}{1+\mathrm{e}^{-\left(\mathrm{a}+\beta_{1} \mathrm{x}_{1}+\beta_{2} \mathrm{x}_{2}+\cdots+\beta_{\mathrm{k}} \mathrm{x}_{\mathrm{k}}\right)}}
\end{aligned}
$$

Extending the logic of the simple logistic regression to multiple predictors, one may construct a complex logistic regression as:

$$
\operatorname{logit}(\mathrm{y})=\ln \left(\frac{\mathrm{p}}{1-\mathrm{p}}\right)=a+\beta_{1} \chi_{1}+\beta_{2} \chi_{2}+.+\beta_{k} \chi_{k}
$$

Therefore,

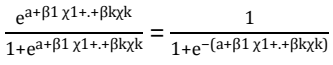

Deriving from the formula given, odds of an event are the ratio of the probability that an event will occur to the probability that it will not occur. If the probability of an event occurring is $p$, the probability of the event not occurring is $(1-p)$. Then the corresponding odds is a value given by:

$$
\begin{gathered}
\text { Odds of }\{\text { Event }\}=\frac{\frac{e^{a+\beta_{1} x_{1}+\beta_{2} x_{2}+\cdots+\beta_{k} x_{k}}}{1+e^{a+\beta_{1} x_{1}+\beta_{2} x_{2}+\cdots+\beta_{k} x_{k}}}}{1-\frac{e^{a+\beta_{1} x_{1}+\beta_{2} x_{2}+\cdots+\beta_{k} x_{k}}}{1+e^{a+\beta_{1} x_{1}+\beta_{2} x_{2}+\cdots+\beta_{k} x_{k}}}}=\frac{e^{a+\beta_{1} x_{1}+\beta_{2} x_{2}+\cdots+\beta_{k} x_{k}}}{1+e^{a+\beta_{1} x_{1}+\beta_{2} x_{2}+\cdots+\beta_{k} x_{k}}} \cdot \\
\frac{1+e^{a+\beta_{1} x_{1}+\beta_{2} x_{2}+\cdots+\beta_{k} x_{k}}}{1} \\
=e^{a+\beta_{1} x_{1}+\beta_{2} x_{2}+\cdots+\beta_{k} x_{k}}
\end{gathered}
$$

Logistic regression calculates the probability of an event occurring

\begin{tabular}{|c|c|c|c|c|}
\hline Category of patients according to diagnosis & Number of individuals & Male & Female & Leads to death \\
\hline ESRD & $148(59.9 \%)$ & $78(52.70 \%)$ & $70(47.30 \%)$ & $6(4.05 \%)$ \\
\hline ESRD with Pulmonary Congestion & $45(18.22 \%)$ & $21(46.7 \%)$ & $24(53.3 \%)$ & $26(9 \%)$ \\
\hline ESRD with Cardiovascular Disease & $54(21.86 \%)$ & $22(40.7 \%)$ & $32(59.3 \%)$ & $37(12.8 \%)$ \\
\hline Total & 247 & 121 & 126 & 69 \\
\hline
\end{tabular}
over the probability of an event not occurring, the impact of independent variables is usually explained in terms of odds. With logistic regression, the mean of the response variable $p$ in terms of an explanatory variable $x$ is modeled relating $p$ and $x$ through the equation $p=\alpha+\beta_{1} X_{1}+\beta_{2} X_{2+\ldots} \beta_{k} X_{k}$.

Table 1: Profile risk of ESRD patients 


\section{RESULTS AND DISCUSSION}

Risk profile of patients with ESRD from M. V makabali memorial hospital

Then, if two events are compared, for example, the first event (odds ${ }_{1}$ ), where a dice rolled would come out 3 since its odds is 1:6 or the dice would come out with 3 dots, once most likely, of the total 6 possible outcomes, compared to the another event (odds 2 ), the chances of the dice to come out as an even number which is 3 times most likely to come out from a total of 6 possible outcomes.

$$
\text { It would be, } \frac{\text { odds } 1}{\text { odds }} \text {. }
$$

Thus, it can be derived from the equation, the comparison of two events whether the first event will be likely to happen than another event or vice versa [7].

Based on table 1, out of 247 ESRD patients of M. V. Makabali Memorial Hospital, 148 individuals (59.9\%) have ESRD without complications, 78 or $53.79 \%$ of it are female, and 70 individuals $(48.61 \%)$ consist of the male. Also, out of 148 patients, 6 individuals or $2.1 \%$ died because of the disease. 45 patients (18.22\%) have pulmonary congestions, 21 patients (46.7\%) are male and 24 patients (53.3\%) are female. Because of the complication, 26 patients $(9 \%)$ died of pulmonary congestions. while 54 (21.86\%) ESRD patients have the complication of cardiovascular disease. There are more female ESRD patients diagnosed with cardiovascular disease $(59.3 \%)$ than male patients with (40.7\%), and 37 patients (12.8\%) died because of the disease. Table 1 also shows that more patients have the complication of cardiovascular disease than pulmonary congestions. It also indicates that more patients died because of the complication of the cardiovascular disease.

According to the review article by Rika Jimbo and Tatsuo Shimosawa entitled "Cardiovascular Risk Factors and Chronic Kidney DiseaseFGF23: A Key Molecule in the Cardiovascular Disease", cardiovascular disease is a major risk factor for Chronic kidney disease (CKD) and might possibly lead to premature death. Investigators produced a conclusive report from global prevalence and absolute burden of CKD in 2010 by pooling data from population-based studies. They explored MEDLINE (January 1990 to December 2014) and found that CKD is a global health challenge especially in low-and-middle-income countries. National and international efforts for prevention, detection, and treatment of CKD are needed to reduce its morbidity and mortality worldwide [8]. Among the variables included in the equation, two are revealed to be significantly increasing as well as their coefficients, which indicates a positive increasing effect on the probability risks on the life of the patient. The coefficients of these two variables also indicate a positive increase to the probability envisioned. However, the rest of the variables: age and gender, appear to be insignificant. It means that the probability of having the risk mentioned does not necessarily increase whenever a person ages and being male or female does not upsurge the chance of dying of an ESRD patient. Thus, these insignificant variables will no longer be included in the equation.

Risk factors that cause to increase the death probability of an ESRD patient

With the use of the forward stepwise method in Logistic Regression Analysis as well as an alpha with a value of 0.001 , the result shows that out of the four covariates, only two variables appear to become significant and these are the Cardiovascular Disease and Pulmonary Congestions with the same p-value of 0.000 . Both factors have positive regression coefficients which signify that whenever a person has an attribute of any of the risk factors, the patient has a higher risk to encounter death.

Table 2: Variables

\begin{tabular}{lll}
\hline Variables & $\boldsymbol{\beta}$ & p-value \\
\hline Cardiovascular Disease & 3.944 & 0.000 \\
Pulmonary Congestions & 3.490 & 0.000 \\
Age & -0.005 & 0.670 \\
Gender & -0.331 & 0.382 \\
Constant & -2.717 & 0.000 \\
\hline
\end{tabular}

Table 3: Significant factors

\begin{tabular}{lll}
\hline Factors & $\boldsymbol{\beta}$ & p-value \\
\hline Cardiovascular Disease & 3.942 & 0.000 \\
Pulmonary Congestion & 3.478 & 0.000 \\
Constant & -3.164 & 0.000 \\
\hline
\end{tabular}

\section{Logistic regression model}

Using only the significant variables and based on the coefficients acquired, the logistic regression model of a person's probability of death is written as:

$$
\mathrm{P}\left(\mathrm{Y}=1 \mid \mathrm{X}_{1}, \mathrm{X}_{2}\right)=\frac{\exp \left(-3.164+3.942 \mathrm{X}_{1}+3.478 \mathrm{X}_{2}\right)}{1+\exp \left(-3.164+3.942 \mathrm{X}_{1}+3.478 \mathrm{X}_{2}\right)}
$$

Where the following variables are labeled as:

$$
\mathrm{X}_{1}=\text { Cardiovascular Disease } \mathrm{X}_{2}=\text { Pulmonary Congestion }
$$

Table 4: ESRD patients with no other complications

\begin{tabular}{ll}
\hline Odds & Probabilities \\
\hline 0.042 & $4.05 \%$ \\
\hline
\end{tabular}

\section{Odds and probabilities of ESRD patients}

An End-Stage Renal Disease carrier patient without any other complication, as the results reveal, has 0.042 odds risk and a smaller probability of $4.05 \%$. This means a patient without any other complications has the lowest further probability risk of death since an End Stage Renal Disease Patient is already inclined to having a risk to suffer death because ESRD already has been a remarkably high risk in a patient's life.

Table 5: ESRD Patients with risk factors

\begin{tabular}{lll}
\hline Risk factors & Odds & Probabilities \\
\hline Cardiovascular Disease & 2.178 & $68.52 \%$ \\
Pulmonary Congestions & 1.37 & $57.79 \%$ \\
Cardiovascular Disease and Pulmonary & 70.53 & $98.6 \%$ \\
Congestions & & \\
\hline
\end{tabular}

\section{Odds and probability of ESRD patients with risk factors}

The odds of ESRD patient with cardiovascular disease is $\mathrm{e}^{-3.164+3.9421 \mathrm{X}_{1}}=2.178$ and the probability of an ESRD patient with a cardiovascular disease to encounter death is:

$$
\frac{\exp \left(-3.164+3.942 \mathrm{X}_{1}\right)}{1+\exp \left(-3.164+3.942 \mathrm{X}_{1}\right)}=68.52 \%
$$

Based on the findings of the research entitled "Cardiovascular Disease in Patients with End-Stage Renal Disease on Hemodialysis in a Developing Country", cardiovascular disease is the main cause of death among patients with end-stage renal disease (ESRD). The study has been undertaken to identify the main cardiovascular diseases and their risk factors in 160 patients with ESRD on hemodialysis (HD) in Brazil. The study shows that the most prevalent cardiovascular diseases in patients with ESRD were left ventricular hypertrophy, atherosclerotic disease, vascular disease and coronary artery disease. Hypertension and dyslipidemia are the common risk factors associated with cardiovascular diseases [9].

And the odds of a patient with pulmonary congestions is $\mathrm{e}^{-3.164+3.478 \mathrm{X}_{2}}=1.37$, which means a person which is already susceptible, 1 times more likely to die if the ESRD patient is diagnosed with Pulmonary Congestion and the probability of an ESRD patient to encounter death is

$$
\frac{\exp \left(\left(-3.164+3.478 \mathrm{X}_{2}\right)\right.}{1+\exp \left(-3.164+3.478 \mathrm{X}_{2}\right)}=57.79 \% \text {. }
$$


About $60 \%$ of ESRD patients displayed moderate-severe lung congestion and this alteration is frequently asymptomatic based on the findings of Zoccali et al. (2013). Lung congestion is reduced but not abolished by ultrafiltration dialysis, and about one third to one fourth of patients still have excessive lung water after dialysis. Lung water in HD patients correlates in an inverse fashion with echocardiography parameters of the systolic and diastolic function, but it is only weakly related with hydration status measured by bioimpedance analysis. Moderate-severe lung congestion is a strong predictor of death and cardiovascular events [10]

It is said in a research last 2012 entitled "chronic kidney disease and risk of mortality, cardiovascular events or end-stage renal disease in older patients with hypertension" that Older individuals with a diagnosis of chronic kidney disease are at higher risk of all-cause mortality, cardiovascular (CV) events and end-stage renal disease.... Until future studies better establish the boundaries of 'normal' glomerular filtration rate in older people, physicians should not underestimate the importance of proteinuria and accurate glomerular filtration rate measurement in choosing the most appropriate antihypertensive combination in daily clinical practice, particularly in patients at high risk of progression to end-stage renal disease [11].

However, if a patient suffers from both complications (cardiovascular disease and pulmonary congestions) his/her odds is [7. ${ }^{3.164+3.942 \square_{1}+3.478 \square_{2}}=70.53 \%$ also, the patient has a higher risk of death with a probability of

$$
\frac{\exp \left(1-3.164+3.942 \mathrm{X}_{1}+3.478 \mathrm{X}_{2}\right)}{1+\exp \left(-3.164+3.942 \mathrm{X}_{1}+3.478 \mathrm{X}_{2}\right)}=98.6 \%
$$

Table 6: Odds ratio of ESRD patient if diagnosed with pulmonary congestion

\begin{tabular}{ll}
\hline Covariate & Odds ratio \\
\hline Pulmonary Congestion & 32.39 \\
\hline
\end{tabular}

\section{Odds Ratios of the covariates}

The table shows the compared value of two odds having a different situation. Comparing the two odds generated from the derived equation, the odds of the ESRD patient divided by the odds of ESRD patient with no other complication, $\frac{\text { odd } s_{\text {pulmunarycongestive }}}{\text { odds } s_{\text {non-pulmonary congestive }}}=\frac{\mathbf{1 . 3 7}}{\mathbf{0 . 0 4 2}}=\mathbf{3 2 . 3 9}$ which means the pulmonary congestive patient is approximately 32 times more likely to encounter death than that of the ESRD patient.

Table 7: Odds Ratio of ESRD patient if diagnosed with cardiovascular disease

\begin{tabular}{ll}
\hline Covariate & Odds ratio \\
\hline Cardiovascular Disease & 51.52 \\
\hline
\end{tabular}

Comparing the odds of an ESRD patient with Cardiovascular disease to an ESRD patient with no complications, $\frac{\text { odds }_{\text {pulmunary congestive }}}{\text { odds } \text { non-pulmonary congestive }_{\text {pul }}}=$ $\frac{\mathbf{2 . 1 7 8}}{\mathbf{0 . 0 4 2}} \mathbf{5 1 . 5 2}$. Possessing Cardiovascular Disease for an ESRD patient would give them higher risks of encountering death 52 times more likely than a patient with just having End Stage Renal Disease.

The table indicates the comparison of the odds of an ESRD patient with both of the ailments mentioned, namely: Cardio Vascular and Pulmonary Congestion, and the odds of an ESRD patient. It shows the value of the odds ratio which is roughly 1669.03 , indicates that an ESRD possessing both ailments encounter death 1669 times more likely to happen than an ESRD patient with no other complications.

Table 8: Odds ratio of ESRD patient if diagnosed with both cardiovascular disease and pulmonary congestion

\begin{tabular}{ll}
\hline Covariate & Odds ratio \\
\hline Cardiovascular Disease and & 1669.03 \\
Pulmonary Congestion & \\
\hline
\end{tabular}

\section{CONCLUSION}

Logistic Regression was used to determine the factors that highly predict the probability of death if one has End-Stage Renal Disease. Evidently, the findings show that age and gender are statistically insignificant contributions to the death of patients with ESRD. It means that getting old or being classified by gender does not necessarily gain risk of experiencing death. Results also show that cardiovascular disease and pulmonary congestions are significant factors of ESRD and cardiovascular disease is the most prevalent risk factor that increases the patients' probability to encounter death. A patient without any complication has a lesser risk of death compared to a patient that has complications. An ESRD patient has only 4.05\% probability to suffer death. ESRD patients with cardiovascular disease have a higher probability of suffering death, than an ESRD patient with pulmonary congestions. A patient with cardiovascular disease has $68.52 \%$ probability to suffer death and an ESRD patient with pulmonary congestion has a $57.79 \%$ probability of dying. If a patient with End-Stage Renal Disease suffers from both pulmonary congestion and cardiovascular disease has a high risk of death with a probability of $98.6 \%$. Finally, because all variables have positive regression coefficients, this study concludes that the higher the number of risk factors the individual has, the higher the risk of death.

ESRD is a serious chronic kidney condition that has a notable impact on the lives of the patients. It is important to understand the experiences of the patients for the effectiveness of their healthcare. This kidney problem must be monitored for the prevention of the development of complications. Evidently, this paper concluded that cardiovascular disease is a risk factor of ESRD that shows a higher probability of a patient to encounter death. Moreover, patients must be aware of their status to avoid the development of cardiovascular disease.

Because of the nutritional status of ESRD patients, it is highly recommended to assess their health condition and must maintain a healthy lifestyle and appropriate medication. Also, ESRD patients should also know what the foods that are shouldn't be eaten, by which they should avoid high-sodium diets. Nutrition counseling should be intensive initially and provided every 1 or 2 mo thereafter. If nutrient intake appears inadequate, malnutrition is apparent or adverse events, or illnesses threaten nutritional status, counseling should be increased.

\section{CONFLICT OF INTERESTS}

Declared none

\section{REFERENCES}

1. Crisostomo S. DOH: Cases of kidney failure on the rise. The Philippine Star. Available from: http://www.philstar.com/ headlines/2014/02/25/1294271/doh-cases-kidney-failurerise. [Last accessed on 25 Feb 2014].

2. Diseases and Conditions: Chronic Kidney Disease. Available from: http://www.mayoclinic.org/diseases-conditions/kidneydisease/basics/complications/con-20026778. [Last accessed on 30 Jan 2015].

3. Foley RN, Parfrey PS. Cardiovascular disease and mortality in ESRD. Available from: http://www.ncbi.nlm.nih.gov/pubmed/ 9831236. [Last accessed on 30 Jan 2015].

4. Preventing Pulmonary Edema in ESRD on Dialysis; 2014. Available from: http://www.kidneyfailureweb.comrespiratorysystem-symptoms/1665.html\#6d. [Last accessed on $30 \mathrm{Jan}$ 2015].

5. Australian Institute of Health and Wellfare. (n.d.). End-stage Kidney Disease. Available from: http://www.aihw.gov.au/ ckd/end-stage-kidney-disease/. [Last accessed on 30 Jan 2015].

6. De La Cruz R. Kidney disease PH's $7^{\text {th }}$. Leading Cause of Death. The Manila Times. Available from: http://www.manilatimes. net/kidney-disease-phs-7th-leading-cause-of-death/77716/. [Last accessed on $22 \mathrm{Feb} 2014$ ].

7. Park H. An Introduction to Logistic Regression: From Basic Concepts to Interpretation with Particular Attention to Nursing Domain; 2013. Available from: http://synapse.koreamed. org/Synapse/Data/PDFData/0006JKAN/jkan-43-154.pdf. [Last accessed on 30 Jan 2014]. 
8. R Jimbo, T Shimosawa. Cardiovascular risk factors and chronic kidney disease-FGF23: A Key Molecule in the Cardiovascular Disease. Int J Hypertension; 2014. Available from: http://www.hindawi.com/journals/ijhy/2014/381082/. [Last accessed on 30 Jan 2014].

9. The Effect of Increasing Age on the Prognosis of Non-Dialysis Patients with Chronic Kidney Disease Receiving Stable Nephrology Care. Available from: http://www.nature.com /ki/journal/ v82/n4/full/ki2012174a.html. [Last accessed on 30 Jan 2015].

10. Myocardial Microvascular Disease and Major Adverse Cardiovascular Events in Patients with End-Stage Renal Disease: Rationale and Design of the Microcard Study". Available from: http://ndt.oxfordjournals.org/content/27/7/
2886. abstract?sid=dfc10f6f-ac6d-4b9f-bc57-0d8f0c010857. [Last accessed on 30 Jan 2015].

11. Silva LS, Oliveira RA, Silva GB, Lima JW, Silva RP, Liborio AB, et al Cardiovascular Disease in Patients with End-Stage Renal Disease on Hemodialysis In A Developing Country; 2012. Available from http://www.ncbi.nlm.nih.gov/pubmed/22382216. [Last accessed on 30 Jan 2012].

\section{How to cite this article}

- Jackie D Urrutia, Jaya C DE Guzman, Aaron Vito M Baygan, Edcon B Baccay, Lincoln A Bautista. Probability estimation on the risk of mortality of patients with the end-stage renal disease. Int J Pharm Pharm Sci 2016;8 Suppl 2:1-5. 